\title{
Estudio histopatológico e inmunohistoquímico de páncreas en perros diabéticos inducidos con aloxano
}

\author{
Histopathologic and immunohistochemical study of pancreas \\ in alloxan-induced diabetic dogs \\ V Cubillos*, C López, A Alberdi \\ Instituto de Patología Animal, Facultad de Ciencias Veterinarias, Universidad Austral de Chile, Valdivia, Chile.
}

\begin{abstract}
SUMMARY
The aim of this study was to evaluate pancreatic microscopic lesions with the presence or absence of insulin in Langerhans islets at different times post-induction (p.i.) in alloxan-induced diabetic type I dogs. A group of 20 mixed breed, male adult dogs were used, subdivided in 2 groups: Control $(\mathrm{n}=5)$ and Experimental $(\mathrm{n}=15)$. The Experimental Group was subdivided into groups of 3 animals each which were alloxan-induced intravenously $(75 \mathrm{mg} / \mathrm{kg}$ ). Dogs were sacrificed at different times p.i. (2 hours, 24 hours, 7 days, 14 days, 21 days). Pancreatic samples were taken for histopathologic and immunohistochemical study against insulin and Ki-67. The Control Group considered 5 healthy dogs, one for each Experimental Group. Plasmatic evaluation of glucose and fructosamine were measured to confirm diabetes. The diabetic status of the Experimental Group was confirmed by the presence of sustained hyperglycaemia and high concentrations of fructosamine after 7 days p.i. Histopathologic results showed a significant decrease $(\mathrm{P}<0.05)$ in the number Langerhans of islets through time, necrotic and vacuolar cellular changes after 2 hours post-induction and fibrosis in the groups with more time p.i. At 7, 14 and 21 days p.i. it was possible to observe proliferation of ductular cells. Immunohistochemistry showed a decrease in immunomarked cells against insulin in groups with more time after induction, and evidence of ductular proliferation. The results allowed establishing a direct relationship between histopathologic lesions in endocrine pancreas and immunohistochemistry against insulin and also showed the proliferation of pancreatic ducts.
\end{abstract}

Palabras clave: aloxano, diabetes, perros.

Key words: alloxan, diabetes, dogs.

\section{INTRODUCCION}

El avance científico en relación con el conocimiento de la Diabetes mellitus (DM) en medicina humana y veterinaria ha sido posible mediante el uso de modelos animales, como roedores y caninos (Rees y Alcolado 2005), siendo el Aloxano ${ }^{\circledR}$ la droga más utilizada para inducir DM Tipo I experimental. La DM Tipo I, también denominada Diabetes mellitus Dependiente de Insulina (IDDM), se caracteriza por la destrucción de las células $\beta$ de los islotes de Langerhans, conduciendo a una insuficiencia de carácter progresivo y total de insulina. Clínicamente, los animales afectados evidencian hipoinsulinemia e hiperglicemia persistentes y requieren la instauración de terapia insulínica (Boitard y Avner 1991, Hoenig 1995). La DM Tipo I inducida mediante administración de Aloxano $^{\circledR}$ es muy similar a la presentación natural de la enfermedad (Watanabe y col 2004).

El Aloxano ${ }^{\circledR}$ es un compuesto químico, estructuralmente similar a la urea (Mendez y Ramos 1994, Tyberg y col 2001) y posee acción necrosante específica y selectiva sobre las células $\beta$ de los islotes de Langerhans. En relación con la acción de este compuesto a nivel pancreático,

\footnotetext{
Aceptado: 28.11.2007.

\# Financiamiento: Proyecto DID UACh 2002-65.

* Casilla 567, Valdivia, Chile; vcubillo@uach.cl
}

se postulan dos teorías, una describe la interacción de los metabolitos del Aloxano ${ }^{\circledR}$ con el zinc pancreático, responsables de la destrucción de las células beta, mientras que otras observaciones sustentan la teoría de la formación de radicales de oxígeno que desempeñan una función significativa en la acción diabetogénica de esta sustancia (Fleitas y col 2000).

La DM tipo I constituye la forma más frecuente de presentación en perros, afectando entre el 50 y $70 \%$ de los pacientes diabéticos (Hoenig 1995).

El objetivo de la presente investigación fue determinar a través del tiempo el daño histológico del páncreas endocrino y su relación con la presencia de insulina en las células $\beta$ (mediante inmunohistoquímica) en perros con DM Tipo I inducida con Aloxano ${ }^{\circledR}$ en comparación con páncreas de perros clínicamente sanos.

\section{MATERIAL Y METODOS}

El material de la presente investigación estuvo constituido por muestras de páncreas de 20 caninos machos enteros, mestizos, de 4 a 7 años de edad, con peso corporal entre 12 a $17 \mathrm{~kg}$ y condición corporal de 3 , de acuerdo a la escala de Hill's Pet Nutrition. Los perros utilizados fueron obtenidos del Proyecto DID S-2000-58 (Universidad Austral de Chile): "Población canina sometida a un programa de eutanasia: caracterización demográfica, motivos 
de eutanasia, hallazgos anatomopatológicos y parasitológicos e impacto en Salud Pública", cumpliendo con los requisitos estipulados por el Comité "Uso de Animales en Investigación” de la Universidad Austral de Chile.

Los perros fueron divididos en 2 grupos: control $(n=5)$ y experimental $(n=15)$. El grupo experimental fue subdividido en 5 subgrupos de 3 animales cada uno, induciéndose DM mediante la administración e.v. de Aloxano® (Alloxan A 7413; 5,6 dioxiuracil monohidratado; Sigma) en dosis única de $75 \mathrm{mg} / \mathrm{kg}$. Los subgrupos experimentales se sacrificaron con Tiopental Sódico ${ }^{\circledR}$ (Lab. Chile) y solución sobresaturada de sulfato de magnesio, vía endovenosa, a las 2 h, 24 h y 7 d, 14 d y 21 d posinducción (p.i.), tomándose muestras de páncreas de la región de la cola, lugar anatómico donde se encuentra la mayor cantidad de islotes de Langerhans en el perro (Redecker y col 1992). Las muestras se fijaron por 24 horas en formalina tamponada al 10\%. Para cada subgrupo experimental se consideró un perro control.

\section{DETERMINACION DE LAS CONCENTRACIONES PLASMATICAS} DE GLUCOSA Y FRUCTOSAMINA

A fin de confirmar la instauración del estado diabético se procedió a realizar un muestreo seriado (basal, 2 horas, 24 horas, 7 días, 14 días y 21 días p.i.), colectándose $5 \mathrm{ml}$ de sangre de la totalidad de perros a partir de la vena cefálica. $3 \mathrm{ml}$ fueron colectados en tubos con heparina para la determinación de fructosamina en plasma, y los $2 \mathrm{ml}$ restantes en tubos con $\mathrm{NaF}$ para determinar las concentraciones de glucosa plasmática, según los métodos Test de Reducción con Azul Nitrotetrazolio (NBT) (MPR3 Fructosamina 1054686. Lab. Boehringer Mannheim) y GOD/PAD (Cat. $\mathrm{N}^{\circ}$. GL 2623. Lab. Randox), respectivamente.

\section{ESTUDIO MICROSCOPICO}

Para el estudio microscópico del páncreas las muestras fueron laminadas y fijadas en formalina tamponada al $10 \%$ durante 24 horas. Los procesos de deshidratación, aclaración e impregnación de las muestras se realizaron en autotécnico (Shandon Elliot), para luego ser incluidas en parafina a $60{ }^{\circ} \mathrm{C}$. Posteriormente se cortaron a 4 micras con micrótomo de rotación (R. Jung. Ag. Hindenberg), y se tiñeron con hematoxilina-eosina (H/E). El estudio consistió en la determinación de los tipos de lesiones presentes en el páncreas endocrino y en la cuantificación del número de islotes de Langerhans y conductos pancreáticos, para lo cual de cada corte histológico de páncreas teñido con $\mathrm{H} / \mathrm{E}$ se eligieron 10 campos al azar, utilizando un aumento $10 \mathrm{X}$.

\section{INMUNOHISTOQUIMICA (IHQ) Y ESTUDIO MORFOMETRICO}

El estudio de IHQ consistió en la determinación de insulina en las células beta pancreáticas y en la determinación de proliferación celular a nivel de los conductos pancreáticos. Se realizaron cortes seriados de páncreas a 4 micras de espesor, los que fueron montados en portaobjetos positivados Super Frost Plus (Thermo Electron Corporation - Cat No 6776214).

La determinación de insulina en las células $\beta$ se realizó mediante la técnica de Streptavidina-Peroxidasa utilizándose: Ac. Primario policlonal antiinsulina (IgG cobayo) (Zymed $^{\circledR}$, Cat No 18-0067) en dilución 1:800 por 12 horas a $4{ }^{\circ} \mathrm{C}$, Ac. secundario biotinilado (Anti IgG cobayo-cabra) (Vector ${ }^{\circledR}$, Cat $\mathrm{N}^{\circ}$ BA 7000) en dilución 1:500, a $21{ }^{\circ} \mathrm{C}$ por una hora, Complejo Streptavidina-Peroxidasa (Dako ${ }^{\circledR}$ Cat $\mathrm{N}^{\circ} \mathrm{P}$-0397) en dilución 1:500 a $21{ }^{\circ} \mathrm{C}$ por una hora. Para la determinación de proliferación celular a nivel de los conductos pancreáticos se utilizaron Ki-67 (Ac. prediluido, clon MIB-1, immunotech, SA, Marseille), anticuerpo monoclonal que identifica un antígeno nuclear que se encuentra en células en las fases proliferativas del ciclo celular (G1, S, G2 y M), y el kit de detección Ultravision (LabVision Cat. No TP-015-HD). Como control negativo, en ambos casos, se omitió el anticuerpo primario. Para el revelado (insulina y Ki-67) se utilizó DAB (Zymed ${ }^{\circledR}$, Cat $\mathrm{N}^{\mathrm{o}}$ 00-2014) por un minuto y la contratinción se realizó con hematoxilina de Mayer por 30 segundos.

Para el estudio morfométrico se utilizaron cortes histológicos de páncreas inmunomarcados contra insulina de cada animal (Grupos Control y Experimentales). Las imágenes se capturaron a partir de un microscopio Olympus Modelo CX31RBSFA, utilizando para ello una cámara digital Nikon Coolpix 995. Posteriormente se recurrió a un programa informático de análisis de imágenes (ImageProPlus ${ }^{\circledR}$ v. 4.1, Media Cibernetics). En cada uno de los grupos en estudio se determinó el área total de tejido endocrino (islotes de Langerhans) $\left(\mathrm{mm}^{2}\right)$. La determinación del área total de tejido pancreático por grupo correspondió a la sumatoria del área total del tejido presente en 5 campos histológicos de 10X, los cuales fueron seleccionados al azar, a fin de obtener una distribución representativa de los islotes de Langerhans. El área total de tejido endocrino correspondió a la sumatoria de las mediciones individuales del área perimetral de cada uno de los islotes presentes en los 5 campos determinados con anterioridad. El área total de tejido pancreático analizada en el presente estudio se estableció mediante la medición del área total existente en un campo a un aumento de $10 \mathrm{X}\left(546,036 \mathrm{~mm}^{2}\right)$. Dado que se analizó un total de 5 campos por muestra (por animal), el área total analizada fue la sumatoria de los 5 campos $\left(2.730,182 \mathrm{~mm}^{2}\right)$.

\section{ANALISIS ESTADISTICO}

La normalidad de la distribución de los datos se estableció mediante la prueba de Shapiro-Wilks. Tanto para el recuento de los islotes de Langerhans, conductos pancreáticos, como para las concentraciones plasmáticas de glucosa y fructosamina se calcularon los promedios y error estándar para cada grupo en estudio. La significancia de las diferencias en el recuento de islotes de Langerhans y conductos pancreáticos entre los distintos grupos se 
evaluó mediante ANDEVA. Para la comparación de las concentraciones plasmáticas de glucosa y fructosamina en los distintos grupos en estudio se efectuó la prueba de ANDEVA de muestras repetidas. En aquellos casos en que se observaron diferencias significativas se recurrió a la prueba de comparación múltiple de Tukey Krammer, empleando un nivel de significación de 5\%. Los resultados se procesaron en el Programa Stat Graphic Plus v. 2.0, complementado con una planilla Excel 2000. Los resultados fueron expresados como Promedios \pm EE.

\section{RESULTADOS}

En relación con la glucosa y fructosamina plasmáticas, a partir del séptimo día p.i se observó un marcado incremento de las concentraciones, por encima del rango establecido para la especie, las cuales se mantuvieron a través del tiempo. Estos resultados permitieron confirmar la instauración del estado diabético en los perros en estudio. El análisis estadístico reveló diferencias significativas $(\mathrm{P}<0,05)$ entre los valores basales y los obtenidos a los 7, 14 y 21 días p.i. (figuras 1 y 2).

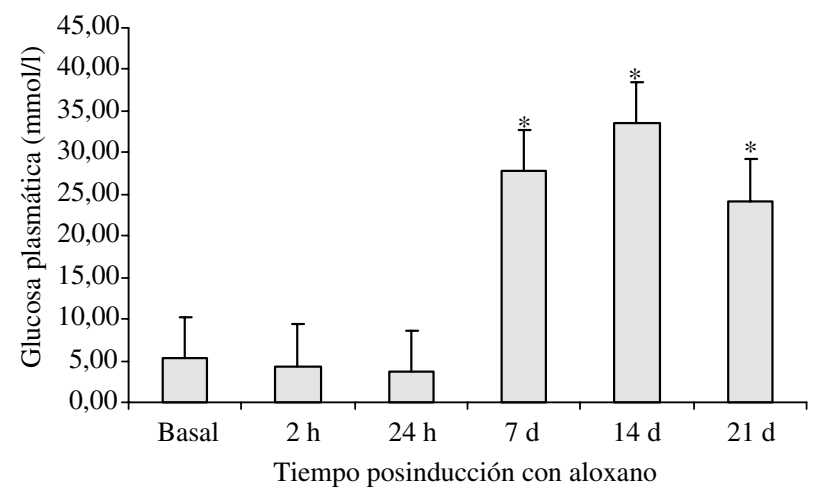

Figura 1. Valores promedio de glucosa plasmática $\pm \mathrm{EE}$ en perros inducidos con Aloxano ${ }^{\circledR}$, de acuerdo al tiempo posinducción. $* \mathrm{P}<0,05$.

Mean values of plasmatic glucose \pm SE in alloxan-induced diabetic dogs, according to the time post-induction. $* \mathrm{P}<0.05$.

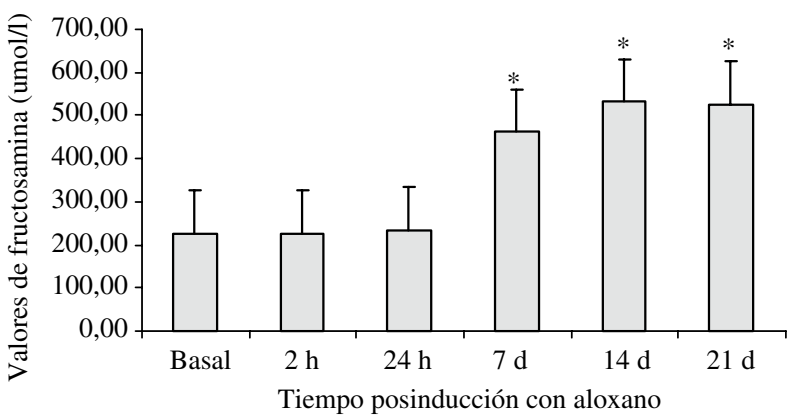

Figura 2. Valores promedio de fructosamina plasmática $\pm \mathrm{EE}$ en perros inducidos con Aloxano ${ }^{\circledR}$, de acuerdo al tiempo posinducción. $* \mathrm{P}<0,05$.

Mean values of plasmatic fructosamine \pm SE in alloxan-induced diabetic dogs, according to the time post-induction. $* \mathrm{P}<0.05$.
ESTUDIO MICROSCOPICO E INMUNOHISTOQUIMICO DE PANCREAS

En relación con el estudio microscópico, el análisis histológico de las muestras con $\mathrm{H} / \mathrm{E}$ permitió establecer diferencias en el tiempo. En el Grupo Control, la observación microscópica por campo 10X permitió establecer un promedio de 8 islotes de Langerhans; en cambio, en los páncreas de los Grupos Experimentales se apreció una disminución gradual, siendo ésta más severa en aquellos Grupos con mayor tiempo p.i. El análisis estadístico mostró diferencias significativas $(\mathrm{P}<0,05)$ entre el Grupo Control y los correspondientes a las $24 \mathrm{~h}, 7 \mathrm{~d}, 14 \mathrm{~d}$ y $21 \mathrm{~d}$ p.i. (figura 3 ).

En relación con los aspectos lesionales, las células de los islotes de Langerhans evidenciaron picnosis a partir de las 2 horas p.i. (figura 4). A los $7 \mathrm{~d}$ se apreció una menor intensidad en esta condición, siendo escasa a los 14 d y estando ausente a los $21 \mathrm{~d}$. Además, se apreció una estrecha relación entre picnosis y vacuolización de las células de los islotes. Finalmente, en los islotes de los Grupos de 14 y 21 d p.i. se observó fibrosis, la cual se hizo más evidente en el Grupo con mayor tiempo p.i. (21 d). En los ácinos pancreáticos la lesión más significativa la constituyó la proliferación de conductos intra e interlobulares (figura 5), la cual fue evidente en los animales con mayor tiempo p.i. (7, 14 y 21 días p.i. respectivamente) (cuadro 1).

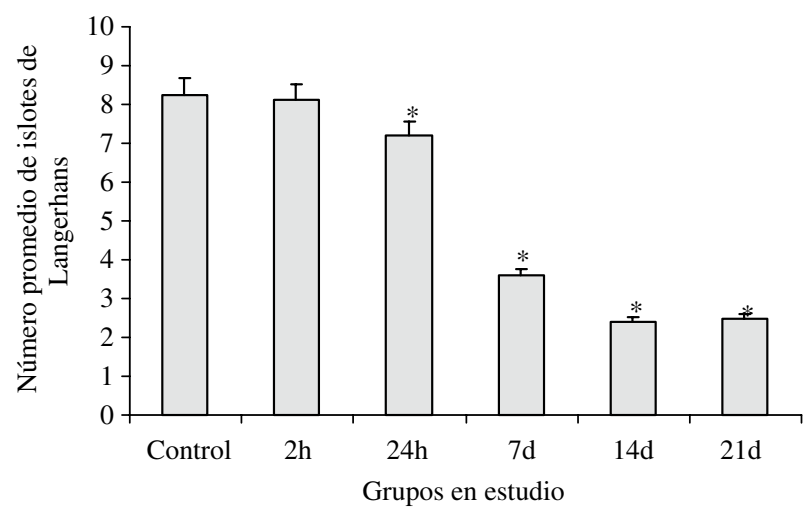

Figura 3. Número promedio de islotes de Langerhans \pm EE en perros Controles e inducidos con Aloxano ${ }^{\circledR}$. $* \mathrm{P}<0,05$.

Mean number of Langerhans islets $\pm \mathrm{SE}$ in control group and alloxan-induced diabetic dogs. $* \mathrm{P}<0.05$.

En relación con la detección de insulina, el estudio inmunohistoquímico permitió observar marcadas diferencias de inmunomarcación entre el Grupo Control y los Experimentales. Es así como en los perros del Grupo Control la totalidad de los páncreas presentaron inmunomarcación positiva intensa de las células $\beta$ en el interior de los islotes de Langerhans (figura 6), la cual también se observó en los Grupos de 2 y 24 horas p.i. Sin embargo, en los páncreas de los Grupos de 7, 14 y 21 días p.i. la inmunomarcación 


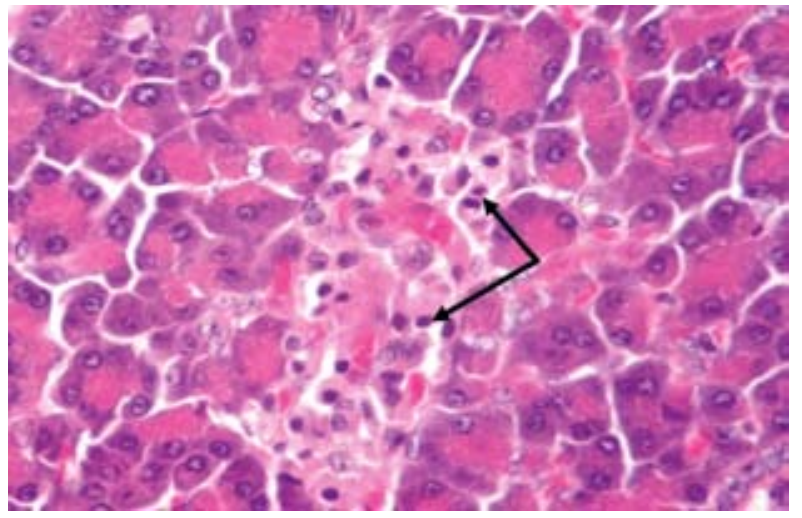

Figura 4. Núcleos picnóticos en islote de Langerhans ( $\uparrow) 24$ horas p.i. con Aloxano ${ }^{\circledR}$. H/E 400 X.

Picnotic nuclei in a Langerhans islet $(\uparrow) 24$ hours after alloxan induction. H/E $400 \mathrm{X}$.

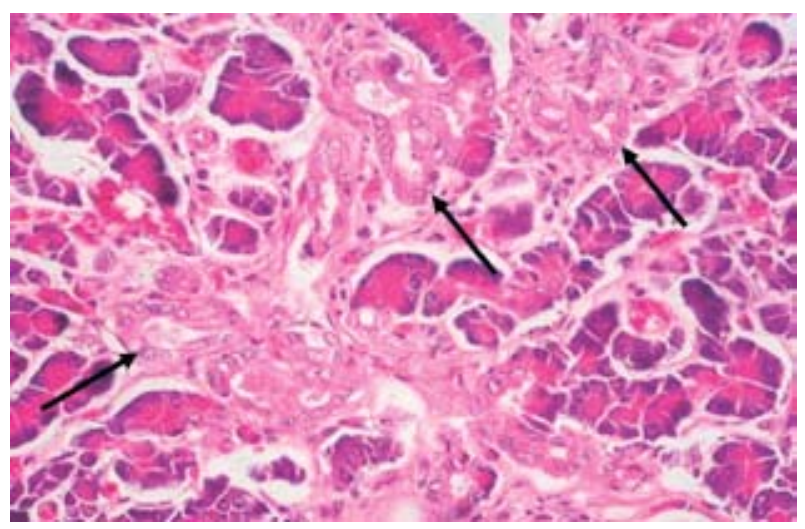

Figura 5. Proliferación de conductos en páncreas de perro. 21 días p.i. con Aloxano $^{\circledR}$ ( $\left.\uparrow\right)$. H/E 400 X. induction $(\uparrow)$. H/E $400 \mathrm{X}$.

Duct proliferation in pancreas dog. 21 days after alloxan-

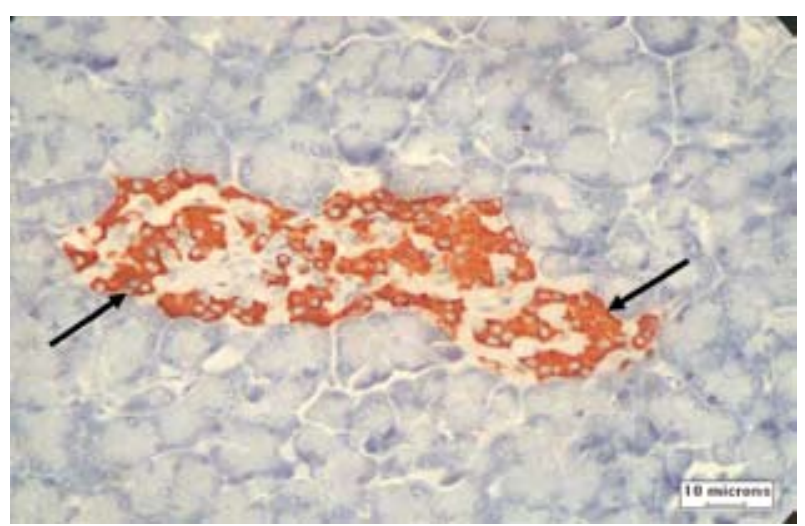

Figura 6. Células $\beta$ inmunomarcadas en islote de Langerhans ( $)$ (grupo control). Streptavidina-Peroxidasa. $400 \mathrm{X}$.

Immunomarked $\beta$ cells in a Langerhans islet (control group). Streptavidine-Peroxidase. $400 \mathrm{X}$.

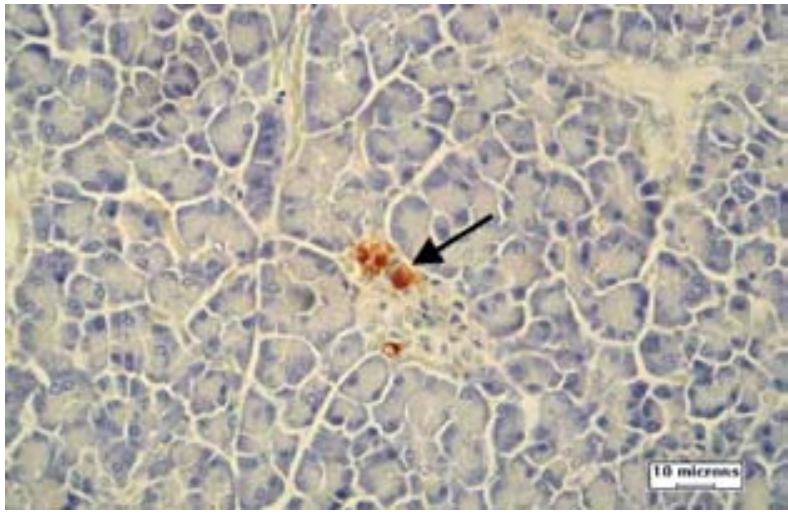

Figura 7. Células $\beta$ inmunomarcadas en islote de Langerhans ( $\uparrow$ ) de perro diabético (7 días p.i.). Streptavidina-Peroxidasa. $400 \mathrm{X}$.

Immunomarked $\beta$ cells in a Langerhans islet of a diabetic
$\operatorname{dog}(7$ days post-induction). Streptavidine-Peroxidase. $400 \mathrm{X}$.

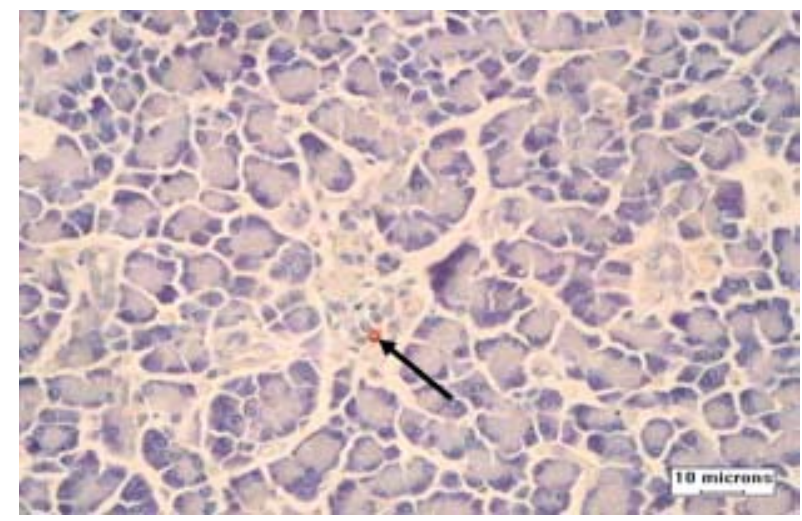

Figura 8. Célula $\beta$ inmunomarcada en islote de Langerhans ( $\uparrow$ ) de perro diabético (21 días p.i.). Streptavidina-Peroxidasa. $400 \mathrm{X}$.

Immunomarked $\beta$ cell in a Langerhans islet of a diabetic $\operatorname{dog}$ (21 days post-induction). Streptavidine-Peroxidase. $400 \mathrm{X}$.

se redujo sustantivamente, observándose una disminución en su intensidad como en el número de células inmunomarcadas dentro de cada islote (figuras 7 y 8 ).

El estudio inmunohistoquímico contra Ki-67 permitió establecer actividad proliferativa a nivel de los conductos pancreáticos a los 7, 14 y 21 días p.i., evidenciándose a nivel de las células epiteliales ductales intensa inmunomarcación nuclear en comparación con el Grupo Control.

\section{ESTUDIO MORFOMETRICO}

Los resultados en relación con el área total de tejido endocrino $\left(\mathrm{mm}^{2}\right)$ en páncreas mostraron diferencias significativas $(\mathrm{P}<0,05)$ a $\operatorname{los} 7,14$ y 21 días p.i., apreciándose una disminución de tejido endocrino en el tiempo (cuadro 2). 
Cuadro 1. Trastornos histológicos en páncreas endocrino de perros controles e inducidos con Aloxano ${ }^{\circledR}$

Histologic lesions in endocrine pancreas of control and alloxan-induced diabetic dogs.

\begin{tabular}{|c|c|c|c|c|c|}
\hline \multirow{3}{*}{ Grupos } & & \multicolumn{4}{|c|}{ Trastornos pancreáticos* } \\
\hline & & \multicolumn{3}{|c|}{ Islotes de Langerhans } & \multirow{2}{*}{$\begin{array}{c}\text { Acinos } \\
\begin{array}{c}\text { Proliferación } \\
\text { conductos }\end{array} \\
\end{array}$} \\
\hline & & Picnosis & Vacuolización & Fibrosis & \\
\hline \multirow{5}{*}{ Control } & 1 & - & - & - & - \\
\hline & 2 & - & - & - & - \\
\hline & 3 & - & - & - & - \\
\hline & 4 & - & - & - & - \\
\hline & 5 & - & - & - & - \\
\hline \multirow{3}{*}{$2 \mathrm{~h}$} & 1 & +++ & + & - & - \\
\hline & 2 & +++ & ++ & - & - \\
\hline & 3 & +++ & ++ & - & - \\
\hline \multirow{3}{*}{$24 \mathrm{~h}$} & 1 & +++ & ++ & - & - \\
\hline & 2 & +++ & - & - & - \\
\hline & 3 & +++ & ++ & - & - \\
\hline \multirow{3}{*}{$7 d$} & 1 & ++ & - & - & ++ \\
\hline & 2 & + & + & - & ++ \\
\hline & 3 & ++ & + & - & + \\
\hline \multirow{3}{*}{$14 \mathrm{~d}$} & 1 & + & - & + & ++ \\
\hline & 2 & + & - & + & - \\
\hline & 3 & - & - & + & ++ \\
\hline \multirow{3}{*}{$21 \mathrm{~d}$} & 1 & - & - & ++ & ++ \\
\hline & 2 & - & - & ++ & ++ \\
\hline & 3 & - & - & ++ & ++ \\
\hline
\end{tabular}

* (-) ausencia, (+) leve, (++) moderada, (+++) intensa.

Cuadro 2. Area total de tejido endocrino $\left(\mathrm{mm}^{2}\right)$ y promedio por grupo, en perros controles e inducidos con Aloxano ${ }^{\circledR}(10 \mathrm{X})$.

Total endocrine area $\left(\mathrm{mm}^{2}\right)$ and mean by group, in control and alloxan-induced diabetic dogs (10X).

\begin{tabular}{|c|c|c|c|c|c|c|}
\hline & \multicolumn{6}{|c|}{ GRUPOS } \\
\hline & $\begin{array}{c}\text { Control } \\
\mathrm{n}=5\end{array}$ & $\begin{array}{c}2 \mathrm{~h} \\
\mathrm{n}=3\end{array}$ & $\begin{array}{c}24 \mathrm{~h} \\
\mathrm{n}=3\end{array}$ & $\begin{array}{c}7 \mathrm{~d} \\
\mathrm{n}=3\end{array}$ & $\begin{array}{c}14 \mathrm{~d} \\
\mathrm{n}=3\end{array}$ & $\begin{array}{c}21 d \\
n=3\end{array}$ \\
\hline \multirow{5}{*}{$\begin{array}{l}\text { Área total de } \\
\text { tejido } \\
\text { endocrino } \\
\left(\mathrm{mm}^{2}\right)\end{array}$} & 39,549 & 30,387 & 39,118 & 5,174 & 4,002 & 3,948 \\
\hline & 38,148 & 35,611 & 32,759 & 3,620 & 3,790 & 2,971 \\
\hline & 43,434 & 42,376 & 24,540 & 3,432 & 3,408 & 4,406 \\
\hline & 28,235 & - & - & - & - & - \\
\hline & 36,575 & - & - & - & - & - \\
\hline PROMEDIO & 37,188 & 36,125 & 32,139 & $4,075 *$ & $3,733 *$ & $3,775 *$ \\
\hline
\end{tabular}

$* \mathrm{P}<0,05$

\section{DISCUSION}

Uno de los principales hallazgos presentes en exámenes de laboratorio clínico en perros diabéticos se relaciona con elevados valores de glicemia $(>10 \mathrm{mmol} / \mathrm{L})$ que se mantienen en forma sostenida a través del tiempo (Hoenig 1995, Gupta y col 2006). Al respecto, en la presente investigación la comprobación de la instauración del estado diabético en el Grupo Experimental se realizó mediante la determinación de hiperglicemia e hiperfructosaminemia sostenidas. Es así como a los 7, 14 y 21 días p.i. se constató marcada hiperglicemia, apreciándose diferencias estadísticamente significativas $(\mathrm{P}<0.05)$ entre los valores basales $(0 \mathrm{~h})$ y aquellas mediciones realizadas a dichos tiempos p.i. (figura 1). Investigaciones realizadas por Black y col (1980) en perros con diabetes inducida experimentalmente observaron hiperglicemia sostenida en el tiempo a partir de las 13 horas p.i. Al respecto, las diferencias observadas entre la presente investigación y Black y col (1980) pueden deberse al hecho de que en la inducción de diabetes dichos autores utilizaron una dosis conjunta de Aloxano ${ }^{\circledR}$ y Estreptozotocina en dosis de $50 \mathrm{mg} / \mathrm{kg}$ y $30 \mathrm{mg} / \mathrm{kg}$, respectivamente. De este modo, es posible que la utilización de ambos fármacos en forma conjunta sea capaz de inducir DM más precozmente, lo cual se refleja en el incremento de las concentraciones de glucosa plasmática a partir de las 13 horas p.i. Por otra parte, Zhao y col (1999), en perros con DM inducida experimentalmente con Aloxano ${ }^{\circledR}$ en dosis de $60 \mathrm{mg} /$ $\mathrm{kg}$, demostraron hiperglicemia sostenida en el tiempo a partir de los 3 días p.i. En el presente estudio los valores plasmáticos de glucosa aumentaron considerablemente a partir de los 7 días p.i., manteniéndose sobre el rango de referencia para la especie (3.0-5.0 mmol/L); sin embargo, dado que el diseño experimental no consideró mediciones entre las $24 \mathrm{~h}$ y los 7 días no es posible establecer el momento exacto en que se presentó el estado de hiperglicemia sostenida.

Si bien la hiperglicemia es una de las principales características de la DM, cabe señalar que no es patognomónica de esta condición. Concentraciones elevadas de glucosa plasmática también pueden observarse en severos episodios de estrés, como igualmente con posterioridad a la ingesta de alimentos (Yamada y col 1993, Mendez y Ramos 1994). En relación con las hormonas del estrés, el cortisol incrementa la gluconeogénesis a nivel hepático, mediante el aumento de la actividad de enzimas responsables de la conversión de aminoácidos en glucosa. La adrenalina guarda relación con el proceso de glucogenólisis hepática, es así como promueve la fosforilación mediante la activación de enzimas responsables de la degradación de la molécula de glucógeno, liberándose glucosa al torrente sanguíneo. Finalmente, la hormona del crecimiento disminuye la captación y utilización de glucosa por las células del organismo, exacerbando la hiperglicemia (Pierluissi y Campbell 1981). Por otra parte, el cortisol y la adrenalina actúan a nivel del tejido muscular promoviendo la glicogenólisis y degradación de proteínas para la liberación de aminoácidos, los cuales sirven de sustrato para la gluconeogénesis (Kitabchi y Murphy 1988). En la presente investigación, la hiperglicemia observada es posible que no sólo haya guardado relación con la destrucción de las células $\beta$, sino que además con el estrés presente en los animales. 
Uno de los métodos que permiten diferenciar entre estados de hiperglicemia sostenida y pasajera se relaciona con mediciones seriadas de glicemia; sin embargo, este procedimiento además de ser engorroso demanda tiempo y muchas veces no es justificable económicamente. La medición de proteínas séricas glicosiladas es otra alternativa para diferenciar la hiperglicemia sostenida de la pasajera. Al respecto, las pruebas de laboratorio más utilizadas se relacionan con la medición de las concentraciones de fructosamina y hemoglobina glicosilada. La fructosamina es una proteína sérica que se forma permanentemente en el cuerpo producto de una reacción no enzimática entre la glucosa y los grupos amino de las proteínas. Debido a que esta reacción es irreversible, su concentración depende principalmente de las concentraciones de glucosa plasmática y de la vida media de las proteínas. En el perro, la proteína a la cual se une la glucosa es la albúmina, la cual tiene una vida media de 1 a 2 semanas. Por esta razón, en gatos y perros la fructosamina se correlaciona con la glucosa presente entre 1 a 2 semanas antes del muestreo (Jensen y col 1992, Kaneko y col 1992, Kawamoto y col 1992). Otra particularidad de esta proteína es que su concentración no se incrementa en cuadros de hiperglicemia de corta duración (Reush y col 1993, Link y Rand 1995, Crenshaw y col 1996). La medición de fructosamina constituye hoy en día una herramienta confiable y de gran utilidad en el monitoreo de la glicemia de perros diabéticos, así como en la respuesta al tratamiento con insulina a través del tiempo (Loste y Marca 2001).

En la presente investigación, las concentraciones plasmáticas de fructosamina evidenciaron un aumento por encima del rango de referencia establecido para la especie a partir de los 7 días p.i. con Aloxano ${ }^{\circledR}$, mostrando diferencias significativas $(\mathrm{P}<0,05)$ entre los valores basales y aquellos obtenidos a los 7, 14 y 21 días p.i., lo cual permite afirmar que las concentraciones plasmáticas de glucosa observadas en los perros en estudio fueron de carácter sostenido.

En cuadros de DM Tipo I, la hiperglicemia sostenida guarda relación directa con la magnitud del daño en el páncreas endocrino, específicamente con la pérdida de células $\beta$ responsables de la producción de insulina. Los resultados del estudio histológico del páncreas evidenciaron una disminución del número de islotes de Langerhans a través del tiempo. Es así como los páncreas de los perros diabéticos sacrificados a las 24 horas y a los 7, 14 y 21 días p.i. presentaron una disminución estadísticamente significativa $(\mathrm{P}<0,05)$ del número de islotes, en comparación con el Grupo Control, observándose una reducción más marcada a partir de los 7 días p.i. Estos resultados están en concordancia con estudios realizados por Rastogi y col (1990) en perros diabéticos inducidos con Aloxano ${ }^{\circledR}$, quienes observaron una significativa disminución del número y del tamaño de los islotes de Langerhans, con posterioridad a la administración de la droga diabetogénica, postulando que "el Aloxano ${ }^{\circledR}$ no sólo es responsable de necrosis de las células $\beta$ sino de la destrucción total de una gran proporción de islotes". Al respecto, en el presente estudio la presencia de cambios necróticos en las células $\beta$ de los islotes de Langerhans se observó a partir de las 2 h p.i. con Aloxano ${ }^{\circledR}$. Este hallazgo está en total concordancia con los estudios realizados por Black y col (1981) en caninos con DM inducida experimentalmente con Aloxano ${ }^{\circledR}$, quienes observaron idénticos cambios en las células $\beta$ a las 2 horas p.i. El Aloxano ${ }^{\circledR}$ se caracteriza en el páncreas por originar necrosis de las células $\beta$ de los islotes de Langerhans mediante la producción de radicales libres, lo cual conduce a una deficiencia absoluta de insulina, originando de esa forma el cuadro de DM (Elsner y col 2002). Estos hallazgos son concordantes con las alteraciones pancreáticas presentes en cuadros de DM Tipo I no experimental, donde la reducción del número y tamaño de los islotes constituye el hallazgo más frecuente, especialmente en cuadros agudos (Taniyama y col 1999). Modelos experimentales en roedores demostraron que la apoptosis de las células $\beta$ del páncreas es el fenómeno final en el desarrollo de la DM Tipo I, estando involucradas en este proceso múltiples moléculas relacionadas con estrés oxidativo como el óxido nítrico (NO) y las especies reactivas de oxígeno (ROS) (Rabinovitch y col 1992, Welsh y col 1994). Existen marcadores tisulares de estrés oxidativo, dentro de los cuales el más utilizado a través de técnicas inmunohistoquímicas en páncreas es el 4-Hidroxi-2-Nonenal (4-HNE), aldehído generado durante la peroxidación de los ácidos grasos poliinsaturados a nivel de la membrana celular (Takatori y col 2003).

Estudios realizados por Imamura y col (1988) en perros con DM inducida mediante pancreatotomía parcial (39-52\% del páncreas) y administración endovenosa de glucosa demostraron una significativa disminución de los islotes de Langerhans en el remanente pancreático. En dicho estudio no se evidenció insulina en los islotes mediante inmunofluorescencia, concluyendo que la depleción de las células $\beta$ se debió a la hiperglicemia. Por otra parte, Unger y Grundy (1985) demostraron que la hiperglicemia crónica en cuadros de DM no controlados origina también depleción de las células $\beta$. En la presente investigación los perros inducidos experimentalmente evidenciaron a partir de los 7 días p.i. hiperglicemia sostenida hasta el final del período experimental (21 días p.i.). En virtud de los antecedentes presentados y de los hallazgos microscópicos, se concluye que la marcada disminución en el número de islotes a partir del $7^{\circ}$ día p.i. puede relacionarse por una parte con el efecto tóxico que origina la hiperglicemia a nivel de los islotes pancreáticos, como asimismo debido a la acción directa del Aloxano ${ }^{\circledR}$, lo cual histológicamente se refleja en una disminución precoz de su número.

Black y col (1980), mediante microscopia electrónica en páncreas de perros con DM inducida con Aloxano ${ }^{\circledR}$, encontraron alteraciones en las células $\beta$ a partir de las 2 horas p.i. con Aloxano ${ }^{\circledR}$, observando picnosis nuclear, vacuolización de las mitocondrias y dilatación del retículo 
endoplásmico rugoso como las primeras alteraciones; sin embargo, a nivel citoplasmático, la degranulación de los gránulos de insulina no es concomitante con la picnosis. De este modo, es posible concluir que si bien el Aloxano ${ }^{\circledR}$ es responsable de picnosis de las células $\beta$ en las primeras 2 horas p.i., la liberación total de insulina a partir de los gránulos intracitoplasmáticos se produce en estadíos posteriores.

Uno de los hallazgos histopatológicos en los páncreas de los perros en estudio fue la proliferación de conductos pancreáticos a partir de los 7 días p.i. con Aloxano ${ }^{\circledR}$. Dicho hallazgo está en concordancia con diversas observaciones realizadas en ratas y ratones a los cuales se les indujo experimentalmente diabetes utilizando diferentes procedimientos (Naya y col 1997, Page y col 2000, Lei y col 2001, Lipsett y Finegood 2002). Al respecto, durante el desarrollo embriológico del páncreas las células endocrinas derivan de células indiferenciadas presentes en el sistema tubuloductal del páncreas (Martin y Capen 1979, Cotran 1995). Además, Teitelman y col (1993) demostraron que las células precursoras de las células $\alpha$ y $\beta$ de los islotes de Langerhans en páncreas de ratones derivan de células epiteliales.

Los hallazgos de Teitelman y col (1993) permitirían explicar los hallazgos realizados en la presente investigación, en el sentido de que la proliferación de células ductales en casos crónicos de DM inducida experimentalmente mediante la necrosis de células $\beta$ se debería a mecanismos compensatorios, en que el organismo como una forma de subsanar la deficiencia de insulina por la destrucción de las células $\beta$ originaría una nueva proliferación de células productoras de insulina a partir de células progenitoras existentes en los epitelios ductales.

Si bien es cierto los estudios de inmunohistoquímica en la presente investigación no mostraron inmunomarcación contra insulina en las células de los conductos pancreáticos en que hubo proliferación, estudios realizados por BonnerWeir (2000) en ratas diabéticas demuestran que la tasa de replicación de condutos pancreáticos es muy elevada y precoz en relación con la neoformación de islotes y ácinos pancreáticos, lo cual indica que la replicación celular tiene lugar antes de la diferenciación. Estos antecedentes permiten explicar la falta de inmunomarcación contra insulina en las células epiteliales de los conductos pancreáticos que proliferaron; en este sentido, estudios de Naya y col (1997) señalan que el proceso de morfogénesis de los islotes en cuadros de deficiencia de insulina constituye una compleja secuencia de cambios celulares y estructurales, los cuales se relacionan con la diferenciación, proliferación y migración de las células endocrinas a partir de células ductales, proceso que finaliza en la formación de nuevos islotes de Langerhans, que contienen células endocrinas diferenciadas. Por otra parte, estudios realizados por Nagoya y col (2004) en perros, posterior a obstrucción parcial de los conductos pancreáticos mediante ligadura, demostraron proliferación de conductos intralobulares a partir de los
15 días. En dicho estudio los autores demostraron que las células presentes en el sistema ductal dieron origen a las 8 semanas a nuevos islotes de Langerhans, los cuales presentaron inmunomarcación positiva contra insulina, concluyendo que los islotes pancreáticos pueden generarse a partir de células precursoras presentes en los conductos pancreáticos. En el presente estudio la inmunomarcación contra Ki-67 permitió evidenciar actividad proliferativa en los conductos pancreáticos a mayor tiempo p.i.

En la presente investigación, un aspecto central lo constituyó la identificación de la insulina en las células $\beta$ de los islotes de Langerhans, con el propósito de relacionar su presencia (o ausencia) con los trastornos histológicos observados con la tinción hematoxilina/eosina, para lo cual se recurrió a inmunohistoquímica contra insulina porcina. Al respecto, Monroe (2005) señala que la insulina porcina es idéntica a la canina estructuralmente, razón por la cual la inmunomarcación de la hormona en las células $\beta$ de los islotes es considerada de carácter específico. Los resultados de la presente investigación permitieron observar importantes diferencias entre el Grupo Control y los Experimentales en relación con las características de la inmumotinción. Es así como a partir de los 7 días p.i. se apreció una importante disminución en la intensidad de la inmunomarcación; de igual forma, en el área inmunomarcada contra insulina en los islotes.

Los hallazgos inmunohistoquímicos y morfométricos observados en la presente investigación son concordantes con estudios realizados por Rastogi y col (1990) quienes mediante inmunoperoxidasa observaron una disminución estadísticamente significativa en el área endocrina de páncreas de perros con diabetes aloxánica, acompañada de una disminución de la inmunomarcación en las células $\beta$ producto de necrosis. Por otra parte, Lima y col (2001) en ratas diabéticas inducidas experimentalmente con Aloxano $^{\circledR}$ también evidenciaron una significativa disminución en el número de islotes, así como en el área inmunomarcada de los mismos a los 15 días p.i. Además, los resultados del estudio morfométrico mostraron una disminución estadísticamente significativa $(\mathrm{P}<0,05)$ del área endocrina en los páncreas de los perros sacrificados a los 7, 14 y 21 días p.i en comparación con el Grupo Control.

Finalmente, los resultados de la presente investigación demuestran la instauración de DM Tipo I en los Grupos Experimentales producto de la administración de Aloxano ${ }^{\circledR}$ en dosis única de $75 \mathrm{mg} / \mathrm{kg}$. Al respecto, clínicamente los perros evidenciaron poliuria, polidipsia y polifagia, además de pérdida de la condición corporal. Por otra parte, las mediciones seriadas de glucosa plasmática permitieron determinar un incremento real en sus concentraciones en los Grupos con mayor tiempo p.i., lo cual está refrendado por el aumento en las concentraciones plasmáticas de fructosamina, proteína glicosilada que permite diferenciar la hiperglicemia pasajera de la sostenida. $\mathrm{Al}$ respecto, las concentraciones plasmáticas de glucosa y fructosamina presentaron un incremento estadísticamente significati- 
vo a partir de los 7 días p.i. con Aloxano $^{\circledR}$, el cual fue de carácter sostenido, característica constante en pacientes diabéticos.

La depleción de las células $\beta$ se hizo evidente a partir de los 7 días p.i.; al respecto, el estudio histológico reveló severos trastornos necróticos en las células de los islotes de Langerhans, acompañados de vacuolización celular a partir de las $2 \mathrm{~h}$ p.i., además de fibrosis en los grupos con mayor tiempo p.i. Dichos hallazgos estuvieron en directa concordancia con la marcada disminución en la inmunomarcación contra insulina a los 7, 14 y 21 días p.i. y con los hallazgos clínicos de hiperglicemia e hiperfructosaminemia en los mismos períodos.

\section{RESUMEN}

El presente estudio se llevó a cabo en perros diabéticos tipo I inducidos con Aloxano ${ }^{\circledR}$, con el propósito de evaluar las lesiones microscópicas presentes en páncreas y relacionarlas con la presencia o ausencia de insulina en los islotes de Langerhans a distintos tiempos posinducción. Se utilizaron 20 caninos mestizos, machos enteros de 4 a 7 años de edad, los cuales fueron divididos en 2 grupos: Control $(n=5)$ y Experimental $(n=15)$. Este último fue subdividido en grupos de 3 animales cada uno y sometido a la inducción de Diabetes mellitus mediante la administración e.v. de Aloxano ${ }^{\circledR}$ en dosis única de $75 \mathrm{mg} / \mathrm{kg}$. Los animales fueron sacrificados a distintos tiempos posinducción (p.i.): 2h, 24h y 7d, 14d, 21d), tomándose muestras de páncreas para examen histopatológico e inmunohistoquímico para determinar insulina y Ki-67. Para cada subgrupo experimental se consideró un perro Control. El estado diabético del Grupo Experimental se comprobó mediante la evaluación de glucosa y fructosamina en plasma. La diabetes fue confirmada por la instauración de hiperglicemia sostenida e hiperfructosaminemia a partir de los 7 días p.i. Los resultados histopatológicos mostraron una disminución significativa $(\mathrm{P}<0,05)$ del número de islotes de Langerhans en el tiempo, acompañada de cambios necróticos y trastornos vacuolares a nivel de las células a partir de las 2 horas p.i. y la presencia de fibrosis en los grupos con mayor tiempo p.i. Además, a los 7, 14 y 21 días p.i. se observó proliferación de los conductos inter e intralobulares a nivel del tejido acinar. Los estudios de inmunohistoquímica y morfométrico mostraron una disminución en la intensidad de inmunomarcación contra insulina, en los grupos con mayor tiempo p.i., así como en el área inmunomarcada contra insulina. Ki-67 permitió dejar en evidencia actividad de proliferación ductal a los 7, 14 y 21 días p.i. Los resultados permitieron establecer una relación directa entre las lesiones histopatológicas en el páncreas endocrino con la inmunomarcación contra insulina y demostrar la proliferación de conductos pancreáticos.

\section{AGRADECIMIENTOS}

Los autores agradecen la colaboración del personal Académico y Técnico Académico del Laboratorio Clínico del Instituto de Ciencias Clínicas Veterinarias -Universidad Austral de Chile-, en particular a la Sra. T.M. Helga Böhmwald por su excelente disposición. Asimismo, se agradece la colaboración del Sr. T.M. Mauricio Castro Iturra, del laboratorio de Anatomía Patológica del Hospital Luis Tisné.

\section{REFERENCIAS}

Black H, IY Roselum, C Capen. 1980. Chemically induced (StreptozotocinAlloxan) diabetes mellitus in the dog. Biochemical and ultrastructural studies. Am J Pathol 98, 295-305.

Boitard C, P Avner, 1991. The immunology of Diabetes mellitus. Nature $351,519$.
Crenshaw K, M Peterson, L Heeb. 1996. Serum fructosamine concentration as an index of glycemia in cats with Diabetes mellitus and stress hyperglycemia. $J$ Vet Intern Med 10, 360-364.

Dunn JS, HL Sheenan, NGB McLeitchie. 1943. Necrosis of islets of Langerhans produced experimentally. Lancet 1, 484-487.

Elsner M, M Tiedge, B Guldbakke, R Munday, S Lenzen. 2002. Importance of the GLUT2 Glucose Transporter for Pancreatic Beta cell Toxicity of Alloxan. Diabetologia 45, 1542-1549.

Fleitas A, R Carballo, G Almeida, AM Quintela, M Alfonso. 2000. Modelo experimental de diabetes en conejos. Rev Cubana Angiol y Circ Vasc $1,10-14$.

Goldner MG, G Gomori. 1943. Alloxan diabetes in the dog. Endocrinology 33, 297-308.

Gupta N, E Park, H Sandhu, T Goh, V Tchipashvili, A Giacca. 2006. Direct and indirect effects of insulin on hepatic glucose production in diabetic depancreatized dogs during euglycemia. $J$ Endocrinol 190, 695-702.

Hoenig M. 1995. Pathophysiology of canine diabetes. Vet Clin N AmSmall 25, 553-561.

Imamura T, M Koffler, JH Helderman, D Prince, R Thirlby, L Inman, RH Unger. 1988. Severe diabetes induced in subtotally depancreatized dogs by sustained hyperglycemia. Diabetes 37, 600-609.

Jensen A, H Houe, C Nielsen. 1992. Critical differences of clinicalchemical parameters in blood from Red Danish dairy cows. Res Vet Sci 52, 86-89

Kaneko J, M Kawamoto, A Heusner, E Feldman. 1992. Evaluation of serum fructosamine concentration as an index of blood glucose control in cats with Diabetes mellitus. Am J Vet Res 53, 1797-1801.

Kawamoto M, J Kaneko, A Heusner, E Feldman, I Koizumi. 1992. Relation of fructosamine to serum protein, albumin, and glucose concentrations in healthy and diabetic dogs. Am J Vet Res 53, 851-855.

Kitabchi AE, MB Murphy. 1988. Diabetic ketoacidosis and hyperosmolar, hyperosmolar, hyperglycaemic nonketotic coma. Med Clin North Am $72,1545-1563$.

Lima L, LC Reis, MA Lima. 2001. Influence of the pineal gland on the physiology, morphometry and morphology of pancreatic islets in rats. Rev Bras Biol 61, 333-340.

Link K, J Rand. 1995. Changes in fructosamine concentration in cats with induction and resolution of chronic hyperglycemia. $J$ Vet Intern Med 9, 180.

Loste A, MC Marca. 2001. Fructosamine and glycated hemoglobin in the assessment of glycaemic control in dogs. Vet Res 32, 55-62.

Mendez DJ, HG Ramos. 1994. Animal models in diabetes research. Arch Med Res 25, 367-375.

Monroe E, D Laxton, EA Fallin, KP Richter, DR Santen, DL Panciera, TL Towell, KA Williams, JR Hart, S Hill, MR Finkler, JS Shinn. 2005. Efficacy and safety of a purified porcine insulin zinc suspension for managing Diabetes mellitus in dogs. J Vet Intern Med 19, 675-682.

Pierluissi J, J Campbell. 1981. Growth hormone and metasomatotrophic diabetes: effects on insulin and proinsulin of serum and pancreas in dogs. Diabetologia 21, 558-562.

Rabinovitch A, WL Suarez, PD Thomas, K Strynadka, I Simpson. 1992. Cytotoxic effects of cytokines on rat islets: evidence for involvement of free radicals and lipid peroxidation. Diabetologia 35, 409-13.

Rastogi S, L Lickey, M Jokay, S Efendic, M Vranic. 1990. Paradoxical reduction in pancreatic glucagon with normalization of somatostatin and decrease in insulin in normoglycemic alloxan-diabetic dogs: a putative mechanism of glucagon irresponsiveness to hypoglycemia. Endocrinology 126, 1096-1104.

Redecker P, A Seipelt, A Jorns, G Bargsten, D Grube. 1992. The microanatomy of canine islets of Langerhans: implications for intra-islet regulation. Anat Embryol (Berl) 185, 131-41.

Reush C, M Liehs, M Hoyer, R Vochezer. 1993. Fructosamine- a new parameter for diagnosis and metabolic control in diabetic dogs and cats. J Vet Intern Med 7, 177-182.

Takatori A, E Ohta, T Inenaga, K Horiuchi, Y Ishii, S Itagaki, S Kyuwa, Y Yoshikawa. 2003. Protective effects of probucol treatment on pan- 
creatic beta-cell function of SZ-induced diabetic APA hamsters. Exp Anim 52, 317-327.

Taniyama H, K Hirayama, Y Kagawa, T Kurosawa, M Tajima, T Yoshino, H Furuoka. 1999. Histopathological and Immunohistochemical analysis of the endocrine and exocrine pancreas in twelve cattle with Insulin-Dependent Diabetes Mellitus (IDDM). $J$ Vet Med Sci 61, 803-810.

Tyrberg B, A Andersson, L Borg. 2001. Species differences in susceptibility of transplanted and cultured pancreatic islets to the beta-cell toxin alloxan. Gen Comp Endocrinol 122, 238-51.

Unger RH, S Grundy. 1985. Hyperglycaemia as an inducer as well as consequence of impaired islet cell function and insulin resistance: implications for the management of diabetes. Diabetologia 28, 119-121.
Watanabe D, H Nakara, Y Yamaguchi, K Akagi, T Hoshiya, Y Nagashima, A Okaniwa, HYoshikawa. 2004. The Pathological Features of Alloxan Diabetes in Beagle Dogs. J Toxicol Pathol 17, 187-195.

Welsh N, DL Eizirik, S Sandler. 1994. Nitric oxide and pancreatic betacell destruction in insulin dependent diabetes mellitus: don't take NO for an answer. Autoimmunity 18, 285-90.

Yamada F, S Inoue, T Sayito, K Tanaka, S Satoh, Y Takamura. 1993. Glucoregulatory hormones in the immobilization stress-induced increase of plasma glucose in fasted and fed rats. Endocrinology 132, 2199-2205.

Zhao G, X Zhang, C Smith, X Xu, M Ochoa, D Greenhouse, T Vogel, CH Curran, TH Hintze. 1999. Reduced coronary NO production in conscious dogs after the development of alloxan-induced diabetes. Am J Physiol 277, 268-278. 
